\title{
Microbiology Susceptibility Reference Identifier
}

National Cancer Institute

\section{Source}

National Cancer Institute. Microbiology Susceptibility Reference Identifier. NCI

Thesaurus. Code C87938.

A character or string used to name, or characterize a microbiology susceptibility reference. 